\title{
Synthesis and biological evaluation of new N-substituted 9-nitro-12,14-dioxo- 9,10-dihydro-9,10-[3,4] epipyrroloanthracen-13-yl derivatives
}

${\text { Maria } \text { Vera }^{1}}^{*}$, James Mc Keown

1 Departamento Sara Noorani Espinardo, 30100 Murcia, España.

${ }^{2}$ School of Pharmacy and Pharmaceutical Sciences, Trinity College Dublin, Trinity Biomedical Sciences Institute (TBSI), 152-160 Pearse Street, Dublin 2, Ireland.

*maria.vera8@um.es

\section{INTRODUCTION}

Cancer is a disease characterised by the uncontrolled growth and spread of abnormal cells, and it is the second leading cause of death globally. The vast majority of patients require chemotherapy in conjunction with surgery or radiological treatments in some steps of their treatment. CLL (Chronic Lymphocytic Leukaemia) is the most common leukaemia in developed countries globally, primarily affecting the elderly. CLL is classed as a clonal disorder of mature B-lymphocytes and its clinical patient prognoses being affected mainly by the mutational status of the Immunoglobulin G Heavy Chain Variable region (IGHV) (with mutated IGHV

holding a better patient prognosis than the wild type variant). Our previous research has demonstrated antiproliferative activity for ethanoanthracene compounds in B-cell lymphomas. In this project we have developed a general procedure for the synthesis of $\mathrm{N}$-substituted 9-nitro-12,14-dioxo-9,10-dihydro9,10-[3,4] epipyrroloanthracen-13-yl derivatives to study their activity against chronic lymphocytic leukaemia cell lines (CLL) and breast cancer.

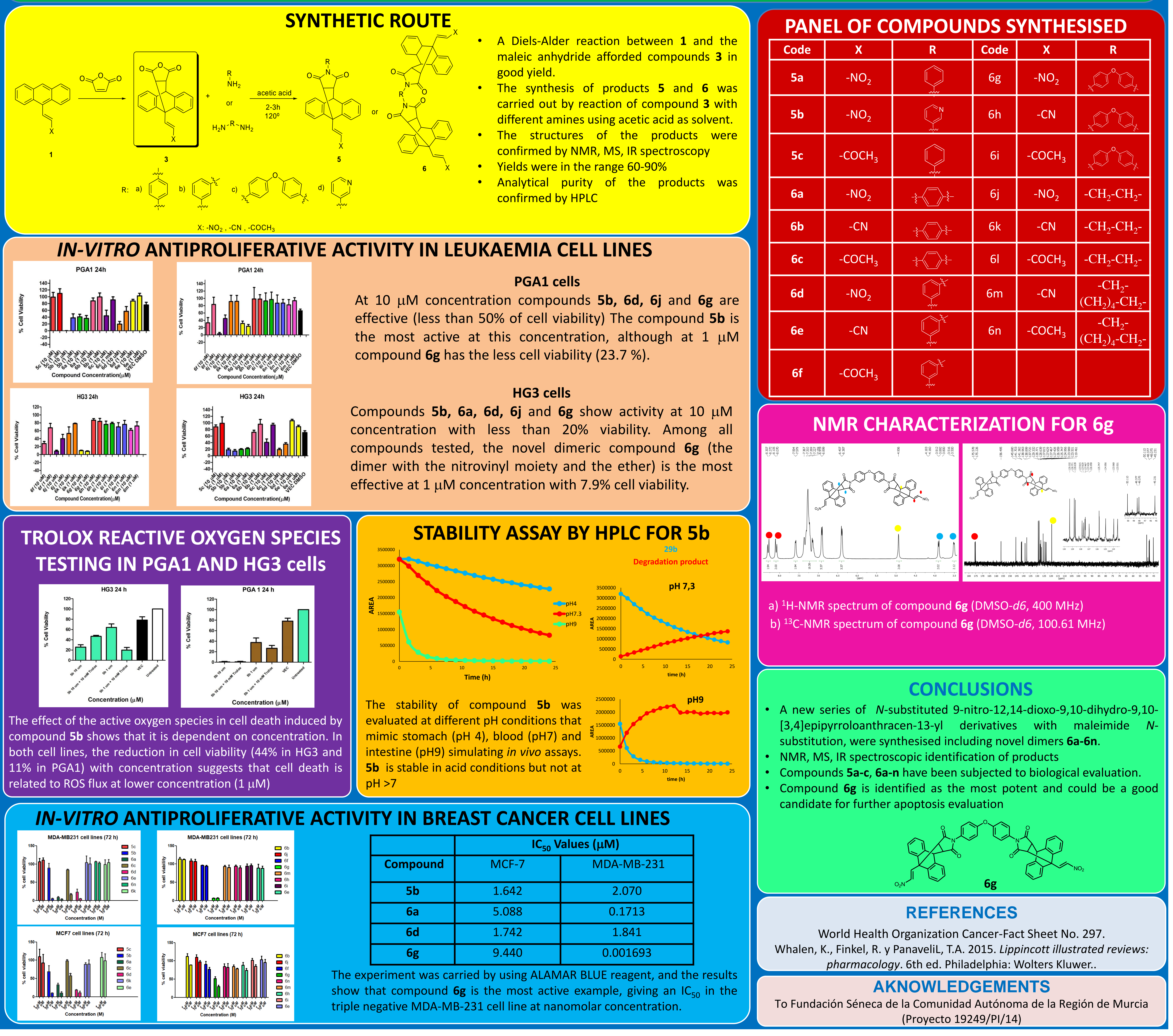

